\title{
Leaf extract of Caesalpinia mimosoides enhances oxidative stress resistance and prolongs lifespan in Caenorhabditis elegans
}

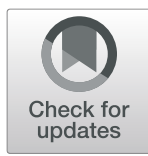

\author{
Panthakarn Rangsinth ${ }^{1,5}$, Anchalee Prasansuklab², Chatrawee Duangjan ${ }^{1,5}$, Xiaojie Gu ${ }^{3,5}$, Krai Meemon ${ }^{4}$
} Michael Wink ${ }^{5^{*}}$ and Tewin Tencomnao ${ }^{6^{*}}$

\begin{abstract}
Background: Caesalpinia mimosoides, a vegetable consumed in Thailand, has been reported to exhibit in vitro antioxidant properties. The in vivo antioxidant and anti-aging activities have not been investigated. The aim of this research was to study the antioxidant activity of C. mimosoides extracts in Caenorhabditis elegans, a widely used model organism in this context.

Methods: C. elegans were treated with C. mimosoides extracts in a various concentrations. To investigate the protective effects of the extract against oxidative stress, wild-type N2 were used to determine survival rate under oxidative stress and intracellular ROS. To study underlying mechanisms, the mutant strains with GFP reporter gene including TJ356, CF1553, EU1 and LD4 were used to study DAF-16, SOD-3, SKN-1 and GST-4 gene, respectively. Lifespan and aging pigment of the worms were also investigated.

Results: A leaf extract of $C$. mimosoides improved resistance to oxidative stress and reduced intracellular ROS accumulation in nematodes. The antioxidant effects were mediated through the DAF-16/FOXO pathway and SOD-3 expression, whereas the expression of SKN-1 and GST-4 were not altered. The extract also prolonged lifespan and decreased aging pigments, while the body length and brood size of the worms were not affected by the extract, indicating low toxicity and excluding dietary restriction.

Conclusions: The results of this study establish the antioxidant activity of $C$. mimosoides extract in vivo and suggest its potential as a dietary supplement and alternative medicine to defend against oxidative stress and aging, which should be investigated in intervention studies.
\end{abstract}

Keywords: Caesalpinia mimosoides, Caenorhabditis elegans, Antioxidant, Oxidative stress, Aging, DAF-16

\section{Background}

It is well established that the consumption of vegetables and fruits is important for human health. Attention has been focused on the effects of bioactive secondary metabolites of plant foods on the prevention of diseases related to oxidative stress. Many secondary metabolites, such as polyphenols, ascorbic acid and carotenoids, can

\footnotetext{
* Correspondence: wink@uni-heidelberg.de; tewin.t@chula.ac.th ${ }^{5}$ Institute of Pharmacy and Molecular Biotechnology, Heidelberg University, Im Neuenheimer Feld 364, 69120 Heidelberg, Germany

${ }^{6}$ Age-Related Inflammation and Degeneration Research Unit, Department of Clinical Chemistry, Faculty of Allied Health Sciences, Chulalongkorn University, Bangkok 10330, Thailand

Full list of author information is available at the end of the article
}

reduce the oxidative stress generated by reactive oxygen species (ROS) $[1,2]$.

ROS are a free radicals that play a role in aging and oxidative stress-related conditions [3]. The production of ROS is a consequence of both exogenous and endogenous factors. Exogenous factors include pollution, ultraviolet radiation and/or unhealthy life habits such as smoking and a diet low in antioxidants [4]. Endogenous factors are related to cellular metabolism, where most of the ROS production occurs during mitochondrial respiration. The antioxidant defense system coevolved along with aerobic metabolism to counteract damage from ROS [5]. When the generation of ROS is not in balance with antioxidant activity, ROS can damage biomolecules,

(c) The Author(s). 2019 Open Access This article is distributed under the terms of the Creative Commons Attribution 4.0 International License (http://creativecommons.org/licenses/by/4.0/), which permits unrestricted use, distribution, and reproduction in any medium, provided you give appropriate credit to the original author(s) and the source, provide a link to the Creative Commons license, and indicate if changes were made. The Creative Commons Public Domain Dedication waiver (http://creativecommons.org/publicdomain/zero/1.0/) applies to the data made available in this article, unless otherwise stated. 
including lipids, proteins and DNA. These activities can mediate aging as well as other chronic diseases such as atherosclerosis, cardiovascular diseases, myocardial infarction, stroke, chronic inflammation, rheumatoid arthritis, cancer, diabetes, septic shock, and other degenerative diseases in humans $[3,6,7]$.

Caesalpinia mimosoides Lam. [recently, reclassified as Hultholia mimosoides (Lam.) E. Gagnon \& G. P. Lewis], a native plant of the northern and northeastern parts of Thailand that is locally called Pak Nam Puya in Thai, also occurs in other areas of tropical eastern Asia. This legume (Fabaceae, subfamily Caesalpinioideae) is a small spiny tropical tree or climbing shrub. Leaves and young twigs of the plant are edible. They are usually consumed in Thailand as a fresh vegetable side dish or appetizer [8]. In addition, the plant has been traditionally used as an anti-flatulent and a remedy against fainting and dizziness. [8] A leaf extract of $C$. mimosoides has also been found to exhibit antioxidant [8], anti-inflammatory [9], anticancer [10] and antimicrobial activities [11]. C. mimosoides has been reported to contain multiple phenolic compounds, including gallic acid and flavonoids which are known antioxidants $[8,12]$. However, in vivo studies of its antioxidant and anti-aging properties have not yet been reported.

Caenorhabditis elegans, a free-living soil nematode, is considered the simplest major multicellular model organism for studying genetic and pharmacological influences on aging and longevity because of its short lifespan, similarities with the human aging process and susceptibility to oxidative stress [13]. Moreover, the genome of C. elegans has been completely sequenced. It carries many homologous genes implicated in human diseases. Major signaling pathways that regulate longevity and stress resistance are well conserved in C. elegans [14-16]. Recent reports suggest that plant extracts with high concentrations of phenolic secondary metabolites exhibit antioxidant and anti-aging activities in C. elegans [17-21].

In this present study, leaves and young twigs of $C$. mimosoides $(\mathrm{CM})$ were exhaustively extracted with hexane, ethyl acetate, and methanol in a Soxhlet apparatus. We selected the methanol extract of CM which exhibited highest polyphenol and flavonoid contents for subsequent experiments. We used this extract to examine in vivo antioxidant and lifespan extension activities in $C$. elegans. Furthermore, the extracts were also investigated to rule out toxicity and a potential influence of dietary restriction.

\section{Methods}

\section{Chemicals}

Dimethyl sulfoxide (DMSO), 2,2'-azino-bis (3-ethylbenzothiazoline-6-sulfonic acid) diammonium salt (ABTS), 2,2-diphenyl-1-picrylhydrazyl (DPPH), Folin-Ciocalteu reagent, quercetin, $5^{\prime}$-fluorodeoxyuridine (FUdR), ampicillin and epigallocatechin gallate (EGCG) were purchased from Sigma-Aldrich (St. Louis, MO, USA). Gallic acid was purchased from TCI America (Portland, OR, USA), L-ascorbic acid from Calbiochem (San Diego, CA, USA), 2,7-dichlorofluorescein diacetate ( $\mathrm{H}_{2}$ DCF-DA) from Fluka Chemie $\mathrm{GmbH}$ (Buchs, Switzerland), juglone (5-hydroxy-1,4-naphthalenedione) from Sigma-Aldrich $\mathrm{GmbH}$ (Steinheim, Germany), and sodium azide from AppliChem GmbH (Darmstadt, Germany). Other reagents used in the extraction process were of analytical grade and purchased from RCI Labscan (Bangkok, Thailand).

\section{Plant material and extraction}

In this study, we used leaves and young twigs, approximately $15-20 \mathrm{~cm}$ in length from the top of the tree shoots of C. mimosoides (CM) collected from the local market in Chiang Rai Province, Thailand. The plant was authenticated and identified with voucher specimen number A014170 (BCU) at the herbarium of Kasin Suvatabhandhu (Department of Botany, Faculty of Science, Chulalongkorn University, Thailand).

Plant material was extracted by the Soxhlet procedure. Briefly, the young twigs and leaves were dried in a ventilated incubator at $40^{\circ} \mathrm{C}$ and ground into a fine powder. Then, approximately $40 \mathrm{~g}$ of the dried powder was uniformly packed into a thimble and sequentially extracted in a Soxhlet apparatus with $400 \mathrm{~mL}$ of three different extracting solvents (hexane, ethyl acetate, and methanol) for at least $24 \mathrm{~h}$ per solvent. The resulting supernatants were collected, filtrated and evaporated to dryness under vacuum. The yields of hexane, ethyl acetate and methanol extraction were 4.02, 6.29 and $29.82 \%(\mathrm{w} / \mathrm{w})$, respectively. Finally, each extract was prepared as a stock solution of $100 \mathrm{mg} / \mathrm{mL}$ in DMSO, sterilized through a $0.2 \mu \mathrm{m}$ pore size syringe filter, stored at $-20^{\circ} \mathrm{C}$, and protected from light until further use.

\section{Quantification of Total phenolic content}

The total phenolic content was determined by the FolinCiocalteu method. The assay was modified for a microplate format as described previously [21-23]. Briefly, $50 \mu \mathrm{L}$ of the extract $(1 \mathrm{mg} / \mathrm{mL})$ was mixed thoroughly with $50 \mu \mathrm{L}$ of a 10 -fold diluted Folin-Ciocalteu reagent. After 20 min incubation, the mixture was neutralized by addition of $50 \mu \mathrm{L}$ of a $7.5 \%(\mathrm{w} / \mathrm{v}) \mathrm{Na}_{2} \mathrm{CO}_{3}$ solution and then kept in the dark at RT for a further $20 \mathrm{~min}$. Finally, the absorbance was measured at $760 \mathrm{~nm}$ using a microplate reader (BioTek Instruments, Winooski, VT, USA). The content of total phenolics was calculated from a standard calibration curve of gallic acid and the results 
were expressed as mg of gallic acid equivalent (GAE) per $\mathrm{g}$ of dry weight extract.

\section{Quantification of flavonoid content}

The total flavonoid content was determined by an aluminum chloride $\left(\mathrm{AlCl}_{3}\right)$ colorimetric method based on the formation of yellow colored aluminum-flavonoid complexes under alkaline condition. The assay was modified for a microplate format, as previously described [21-23]. In brief, $50 \mu \mathrm{L}$ of the extract $(1 \mathrm{mg} / \mathrm{mL})$ was made up to $200 \mu \mathrm{L}$ with $95 \%$ ethanol, and mixed well with $10 \mu \mathrm{L}$ of $10 \%(\mathrm{v} / \mathrm{v}) \mathrm{AlCl}_{3}$ solution and $10 \mu \mathrm{L}$ of $1 \mathrm{M}$ sodium acetate $(\mathrm{NaOAc})$ solution. Then the mixture was allowed to stand for 40 min in the dark and the absorbance was measured at $415 \mathrm{~nm}$ using a microplate reader (BioTek Instruments, Winooski, VT, USA). The content of total flavonoids was calculated from a standard calibration curve of quercetin and the results were expressed as mg of quercetin equivalent (QE) per $g$ of dry weight extract.

\section{DPPH assay}

The 2,2'-diphenyl-1-picrylhydrazyl (DPPH) assay was used to evaluate the free radical scavenging activity of the extract based on its hydrogen atom- or electrondonating capacity to neutralize the stable radical DPPH $(\mathrm{DPPH} \bullet)$, accompanied by a color change from purple to yellow. The assay was performed by using a microplate format. For the assay protocol, $100 \mu \mathrm{LPPH}$ - working solution $(0.2 \mathrm{mM})$ was added to $100 \mu \mathrm{l}$ of the extract at a ratio of $1: 1(\mathrm{v} / \mathrm{v})$. The reaction mixture was incubated in the dark at RT for $30 \mathrm{~min}$, and the absorbance was recorded in a microplate reader (BioTek Instruments, Winooski, VT, USA) at $517 \mathrm{~nm}$. Radical scavenging activity was expressed as the percent inhibition of the DPPH. radicals calculated by the following equation: \% Inhibition $=100 \times$ [Abs of control $-($ Abs of sample - Abs of blank) / Abs of control]. Ascorbic acid (vitamin C) and EGCG were used as controls to study the effective concentration $\left(\mathrm{EC}_{50}\right)$ of the extract.

\section{ABTS assay}

The 2,2' -azinobis-(3-ethylbenzothiazoline-6-sulfonic acid) (ABTS) assay was used to evaluate the free radical scavenging activity of the extract based on its hydrogen atom- or electron-donating capacity to neutralize the stable free radical cation ABTS (ABTS•+), accompanied by a color change from green to colorless. The cation radical ABTS + + working solution was generated by the oxidation of $7 \mathrm{mM}$ ABTS with $2.45 \mathrm{mM}$ potassium persulfate $\left(\mathrm{K}_{2} \mathrm{~S}_{2} \mathrm{O}_{8}\right)$ at a $1: 1(\mathrm{v} / \mathrm{v})$ ratio. The assay was performed by using a microplate format, in which the reaction consisted of $100 \mu \mathrm{l}$ ABTS + working solution and $100 \mu \mathrm{l}$ extracts at a $1: 1(\mathrm{v} / \mathrm{v})$ ratio. The mixture was then incubated in the dark at RT for $45 \mathrm{~min}$, and the absorbance was recorded using a microplate reader (BioTek Instruments) at $734 \mathrm{~nm}$. Radical scavenging activity was expressed as the percent inhibition of the ABTS + radicals calculated by the following equation: \% Inhibition $=100 \times$ [Abs of control - (Abs of sample - Abs of blank) / Abs of control]. Ascorbic acid (vitamin C) and EGCG were used as controls to study the effective concentration $\left(\mathrm{EC}_{50}\right)$ of the extract.

\section{Qualitative phytochemical profiling - LC-MS}

The methanol extract of $C$. mimosoides was submitted to the Institute of Systems Biology (Universiti Kebangsaan Malaysia, Malaysia) to screen for phytochemicals with liquid chromatography-mass spectrometry (LCMS) analysis. The analytical system used was a DionexTM UltiMate 3000 UHPLC system (Thermo Fisher Scientific) coupled with a high-resolution micrOTOF-Q III (Bruker Daltonik GmbH, Bremen, Germany). The chromatographic separation was performed on an AcclaimTM Polar Advantage II C18 column (3 $\mathrm{mm} \times$ $150 \mathrm{~mm}, 3 \mu \mathrm{m}$ particle size) (Thermo Fisher Scientific) with a gradient mobile phase consisting of $0.1 \%$ formic acid in water (solvent $\mathrm{A}$ ) and 100\% acetonitrile (solvent B). The elution program was as follows: $5 \% \mathrm{~B}(0-3 \mathrm{~min})$; $80 \% \mathrm{~B}(3-10 \mathrm{~min}) ; 80 \% \mathrm{~B}(10-15 \mathrm{~min})$ and 5\% B (15-22 $\mathrm{min})$. The flow rate was $400 \mu \mathrm{L} / \mathrm{min}$ within a $22 \mathrm{~min}$ total run time, and the injection volume was $1 \mu \mathrm{L}$. The MS instrument was operated in the positive electrospray ionization (ESI) mode with the following parameters: drying gas flow at $8 \mathrm{~L} / \mathrm{min}$; drying gas temperature at $200{ }^{\circ} \mathrm{C}$; nebulizer pressure at 1.2 bar; capillary voltage at $4500 \mathrm{~V}$; and $\mathrm{m} / \mathrm{z}$ scan range of 50 to 1000 . For identification of putative compounds, the observed (experimental) $\mathrm{m} / \mathrm{z}$ values were compared with the METLIN (CA, USA) and the KNApSAcK (Keyword Search Web Version 1.000.01) databases as well as with the calculated (theoretical) mass values from available previously published data, with an accepted difference of less than $30 \mathrm{ppm}$ (ppm). The relative abundance of a compound is expressed as the percentage of the peak area relative to the total area of all peaks observed in the chromatogram.

\section{Caenorhabditis elegans strains, maintenance, synchronization and treatment}

The C. elegans strains used in this study were wild type N2, BA17 (fem-1(hc17)IV), TJ375 (gpIs1[hsp-16.2:: GFP]), TJ356 (zIs356[daf-16p::daf-16a/b::GFP + rol-6]), CF1553 (mu1s84[pAD76(sod-3::GFP)]), LD1 (ldIs7 [skn1b/c::GFP + rol-6(su1006)]), CL2166 (dvIs19[pAF15(gst4::GFP::NLS)]), and CF1038 (daf-16(mu86)I). The worms were all cultured with nematode growth medium (NGM) containing E. coli OP50 as a food source and 
kept in a $20^{\circ} \mathrm{C}$ incubator. All strains and E. coli OP50 were obtained from Caenorhabditis Genetics Center (CGC), University of Minnesota, USA.

Age synchronization of the worms was achieved by isolating eggs from gravid hermaphrodites. The eggs were prepared by adding lysis solution containing $5 \mathrm{M}$ $\mathrm{NaOH}$ and $5 \% \mathrm{NaOCl}$, followed by vortexing for $10 \mathrm{~min}$ and centrifuging for $2 \mathrm{~min}$ at $1800 \mathrm{rpm}$. Then, the supernatant was removed, and the pellet was washed once in sterile water before centrifugation for an additional 2 min. After discarding water, the remaining eggs were resuspended in M9 buffer for hatching. Larvae were then kept after hatching in S-medium containing E. coli OP50 $\left(\mathrm{OD}_{600}=1.0\right)$. Different treatments were applied according to each experiment. Agar diffusion test was performed to exclude the antimicrobial activity of CM extract against E. coli OP50 (see Additional file 1).

For the experiment, the worms were divided into four groups. The first group was treated with 1\% DMSO (solvent control group). This group served to exclude any toxicity of the solvent used for dissolving the extracts on worms. Groups two through four were treated with 25,50 and $100 \mu \mathrm{g} / \mathrm{ml} \mathrm{CM}$ extracts dissolved in DMSO (maximum 1\%), respectively.

\section{Survival assay under Juglone-induced oxidative stress}

The assay was modified as previously described [19, 24]. Age synchronized L1 larvae stage of wildtype N2 and transgenic CF1038 (DAF-16 loss-of-function mutant) worms were divided into four groups of 80 worms each and treated with different concentrations of $\mathrm{CM}$ extracts or with DMSO diluted in S-medium and bacteria, as mentioned above. After $48 \mathrm{~h}$ of treatment at $20^{\circ} \mathrm{C}$, the pro-oxidant juglone (a naphthoquinone from Juglans regia) was added to a final concentration of $80 \mu \mathrm{M}$, which is a lethal concentration, prior to incubation at $20^{\circ} \mathrm{C}$ for an additional $24 \mathrm{~h}$. Afterwards, surviving and dead worms were counted.

\section{Intracellular ROS accumulation}

Age-synchronized N2 and CF1038 worms (L1 stage) were treated with $\mathrm{CM}$ extracts or DMSO in S-medium at $20^{\circ} \mathrm{C}$ for $48 \mathrm{~h}$. Each group contained $200-300$ individuals. Then, $50 \mu \mathrm{M} \mathrm{H} \mathrm{H}_{2}$ DCF-DA was added and incubated for $1 \mathrm{~h}$ away from light at $20^{\circ} \mathrm{C}$. After that, the worms were mounted on a glass slide and paralyzed by the addition of $10 \mathrm{mM}$ sodium azide, and at least 30 worms were randomly photographed using a fluorescence microscope BIOREVO BZ-9000 with a mercury lamp (Keyence Deutschland GmbH, Neu-Isenburg, Germany) with $\lambda$ ex $480 / 20 \mathrm{~nm}, \lambda$ em $510 / 38 \mathrm{~nm}, 10 \mathrm{X}$ objective lens and constant exposure time. ImageJ software version 1.50i (National Institutes of Health, Bethesda, MD, USA) was then used to measure the relative fluorescence intensity of the full body $[19,24]$.

\section{HSP-16.2 expression}

L1 age-synchronized TJ375 transgenic worms, which express a HSP-16.2::GFP reporter gene, were treated with $\mathrm{CM}$ extracts or DMSO as previously mentioned and incubated at $20^{\circ} \mathrm{C}$ for $72 \mathrm{~h}$. Then, the nematodes were exposed to a nonlethal dose of $20 \mu \mathrm{M}$ juglone for $24 \mathrm{~h}$. The worms were then mounted on a glass slide with $10 \mathrm{mM}$ sodium azide, and images of at least thirty worms per group were taken with a $20 \mathrm{X}$ objective lens at constant exposure time via fluorescence microscopy. Analysis of at least three replicates was performed by quantifying the mean relative fluorescence intensity of the pharynx using ImageJ software [24].

\section{Subcellular DAF-16 localization}

TJ356 transgenic L1 worms, which express DAF-16::GFP fusion protein, were treated with CM extracts or DMSO as previously described and kept at $20^{\circ} \mathrm{C}$. After $72 \mathrm{~h}$, the worms were mounted on a glass slide using $10 \mathrm{mM}$ sodium azide. At least thirty worms per group were imaged on a fluorescence microscope with a $10 \mathrm{X}$ objective lens and constant exposure time. Distribution of the transcription factor DAF-16::GFP in each worm can be in the nucleus, cytoplasm, or the intermediate region between the nucleus and cytoplasm. Worms were sorted and counted according to localization of DAF-16::GFP [19].

\section{Subcellular SKN-1 localization}

LD-1 transgenic worms, which express a GFP reporterfused SKN-1, were age-synchronized at the L1 stage and were treated with CM extracts or DMSO as described previously and kept at $20^{\circ} \mathrm{C}$ for $48 \mathrm{~h}$. Fluorescence intensity was measured by fluorescence microscopy as described above. Then, the worms were mounted on a glass slide using $10 \mathrm{mM}$ sodium azide for paralysis, and at least thirty worms per group were visualized under a fluorescence microscope at a $20 \mathrm{X}$ objective lens and constant exposure time. The transcription factor SKN-1:: GFP in each worm can be located in the nucleus, cytoplasm or the intermediate between the nucleus and cytoplasm. The nematodes were sorted and counted according to the SKN-1::GFP subcellular localization.

\section{SOD-3 expression}

Age-synchronized CF1553 transgenic worms, expressing SOD-3::GFP fusion protein, at the L1 stage were treated with $\mathrm{CM}$ extracts or DMSO as described above and cultured at $20^{\circ} \mathrm{C}$ for $72 \mathrm{~h}$. After treatment, the worms were mounted on a glass slide with $10 \mathrm{mM}$ sodium azide, and at least thirty worms per group were imaged using a fluorescence microscope with a 10X objective lens and 


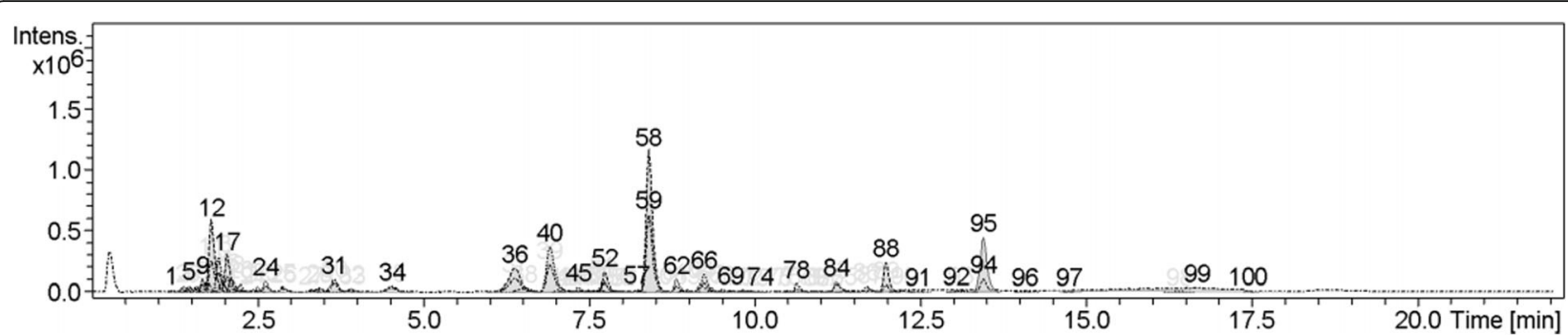

Fig. 1 LC-MS profile of CM methanol extract. The total ion chromatogram (TIC) was generated by LC-MS under positive electrospray ionization. Peak numbers correspond to those in Table 1

constant exposure time. The experiment was repeated at least three times, and analysis was performed by measuring the relative fluorescence intensity using ImageJ software $[19,24]$.

\section{GST-4 expression}

At the L1 stage, synchronized CL2166 worms expressing GST-4::GFP fusion protein were treated with CM extracts or DMSO as described above and kept at $20^{\circ} \mathrm{C}$ for $48 \mathrm{~h}$. After treatment, the worms were exposed to $20 \mu \mathrm{M}$ juglone and incubated at $20^{\circ} \mathrm{C}$ for $24 \mathrm{~h}$. Then, the worms were paralyzed with $10 \mathrm{mM}$ sodium azide on a glass slide, and at least thirty worms were imaged using a fluorescence microscope with a 10X objective lens at constant exposure time. Analysis of three replicates was performed by measuring the relative fluorescence intensity using ImageJ software.

\section{Lipofuscin level}

BA17 transgenic worms, which are thermosensitive and do not lay eggs at $25^{\circ} \mathrm{C}$, were used to measure the expression of lipofuscin, an autofluorescent pigment that accumulates over time and thus is an indicator of aging. The worms at the L1 larval stage were treated with CM extracts or DMSO as mentioned above and cultured at $25^{\circ} \mathrm{C}$ for 16 days. The media and treatments were changed every second day. At day 16, the worms were paralyzed with $10 \mathrm{mM}$ sodium azide on a glass slide, and at least thirty randomly selected worms were imaged on a fluorescence microscope at a 10X objective lens and constant exposure time. Three repeat experiments were performed by measuring the relative fluorescence intensity using ImageJ software.

\section{Longevity assay}

N2 synchronized L4 larvae were plated on NGM agar plates containing a lawn of ampicillin-resistant $E$. coli OP50 supplemented with CM extract at a concentration that exhibited the best antioxidant capacity in the worms. The NGM plates also contained $50 \mu \mathrm{M}$ of $5^{\prime}$ fluorodeoxyuridine (FUdR) to prevent the growth of progeny and $0.1 \mathrm{mg} / \mathrm{mL}$ of ampicillin (Amp) to prevent foreign bacterial contamination. The worms were grown at $25^{\circ} \mathrm{C}$, examined under a stereomicroscope (Nikon Corporation, Tokyo, Japan) and counted daily starting from the first day (day 0) that they were transferred to experimental NGM plates until all individuals had died. The worms were scored as dead when they no longer

Table 1 Proposed phytochemical constituents in methanol extract of C. mimosoides using LC-MS

\begin{tabular}{lllllll}
\hline Peak No. & Rt $(\mathrm{min})$ & {$[\mathrm{M}+\mathrm{H}]^{+}(\mathrm{m} / \mathrm{z})$} & Area $(\%)$ & Proposed compound & Theoretical mass & Mass error (ppm) \\
\hline 12 & 1.8 & 146.0824 & 5.9 & 4-Aminomethylindole & 145.0766 & 9 \\
17 & 2.0 & 256.1181 & 3.0 & N-D-Glucosylarylamine & 255.1107 & 0 \\
36 & 6.4 & 171.0288 & 4.4 & Gallic acid & 170.0215 & 0 \\
40 & 6.9 & 345.0818 & 5.6 & Theogallin & 344.0743 & 0 \\
52 & 7.7 & 329.0869 & 1.6 & Bergenin & 328.0794 & 0 \\
58 & 8.4 & 185.0461 & 13.0 & 3-O-Methylgallate & 184.0372 & 8 \\
59 & 8.4 & 185.0445 & 7.2 & 3-O-Methylgallate & 184.0372 & 0 \\
62 & 8.8 & 481.0979 & 1.1 & Quercetin-3'-glucuronide & 480.0904 & 0 \\
66 & 9.2 & 465.1032 & 1.6 & Quercetin-3-O-glucoside & 464.0955 & 0 \\
88 & 12.0 & 415.2117 & 2.3 & Clausarinol & 414.2042 & 0 \\
95 & 13.4 & 279.1588 & 5.7 & Emmotin A & 278.1518 & 1 \\
\hline
\end{tabular}


Table 2 Total phenolic and flavonoid contents of C. mimosoides extracts

\begin{tabular}{lll}
\hline $\begin{array}{l}\text { Extraction } \\
\text { solvents }\end{array}$ & $\begin{array}{l}\text { Total Phenolic Content } \\
\text { mg GAE/g dry weight extract }\end{array}$ & $\begin{array}{l}\text { Total Flavonoid Content } \\
\text { mg QE/g dry weight extract }\end{array}$ \\
\hline Hexane & $5.35 \pm 0.85$ & $1.76 \pm 0.32$ \\
Ethyl acetate & $323.21 \pm 6.45$ & $8.89 \pm 0.39$ \\
Methanol & $460.25 \pm 3.08$ & $12.55 \pm 0.43$ \\
\hline
\end{tabular}

Values are expressed as the mean \pm SD $(n=3)$

Methanolic extract of CM expressed the highest total phenolic and flavonoid contents when compared to CM extracted by ethyl acetate and hexane

responded to gentle stimulus with a platinum wire and showed no pharyngeal pumping movement. Worms with internally hatched progeny or extruded gonads were censored and excluded from the experiment. The experiment was performed with at least 100 worms per group.

\section{Body length and brood size}

N2 worms were age synchronized by picking adult worms into NGM agar plates with $E$. coli OP50 as a food source. The adult worms were allowed to lay eggs for 2$4 \mathrm{~h}$ before removal, and then the remaining eggs were incubated at $20^{\circ} \mathrm{C}$ for $48 \mathrm{~h}$. After incubation, worms at the L4 larval stage were sorted and used in the experiments. For the body length assay, 50 worms at the L4 larval stage were placed on NGM agar plates supplemented with CM extracts or DMSO in the E. coli OP50 lawn as a food source and cultured at $20^{\circ} \mathrm{C}$ for $24 \mathrm{~h}$. Adult day 1 worms were paralyzed by using $10 \mathrm{mM}$ sodium azide and mounted on a glass slide. At least thirty worms per group were imaged using a $10 \mathrm{X}$ objective lens of bright-field microscope. The software BZ-II Analyzer (Keyence Corp.) was used for the analysis of the body length.

For the brood size assay, each L4 larval stage worm was individually sorted, transferred onto different NGM plates and treated with CM extracts or DMSO. The worms were allowed to grow and lay eggs at $20^{\circ} \mathrm{C}$ and were observed under a dissecting microscope. The eggs were counted and removed to separate them from adult worms every day until the adult worm stopped laying eggs.

Table 3 Free radical scavenging capacities of C. mimosoides extracts

\begin{tabular}{lll}
\hline Samples & \multicolumn{2}{l}{$\mathrm{EC}_{50}$} \\
\cline { 2 - 3 } & DPPH assay & ABTS assay \\
\hline CM methanol $(\mu \mathrm{g} / \mathrm{ml})$ & $8.20 \pm 0.29$ & $5.16 \pm 0.98$ \\
Vitamin $C(\mu \mathrm{M})$ & $40.50 \pm 0.27$ & $26.99 \pm 0.41$ \\
EGCG $(\mu \mathrm{M})$ & $15.56 \pm 0.10$ & $8.95 \pm 0.34$ \\
\hline
\end{tabular}

Values are expressed as the mean \pm SD $(n=3)$

CM methanol extract showed antioxidant properties. $8.20 \pm 0.29$ and $5.16 \pm$ $0.98 \mu \mathrm{g} / \mathrm{ml}$ of the extract were the $\mathrm{EC}_{50}$ to scavenge free radical by DPPH and ABTS assay, respectively

\section{Statistical analyses}

All experiments were performed in at least triplicate. Total phenolic and total flavonoid content as well as $\mathrm{DPPH}$ and ABTS results are presented as the mean \pm standard deviation (SD). The data from $C$. elegans experiments are presented as the mean \pm standard error of the mean (SEM). The differences between groups were analyzed using one-way analysis of variance (ANOVA) followed by Bonferroni's method (post hoc). For the lifespan assay, the statistical significance among different groups was determined by a log-rank (Mantel-Cox) test followed by the Gehan-BreslowWilcoxon test. Differences with $p<0.05$ were considered statistically significant.

\section{Results}

\section{Chemical characterization}

The methanol extract of CM was investigated by LCMS. More than hundred peaks were detected (Fig. 1). Chromatographic peaks were tentatively identified by comparing the MS data with databases based on the search of $\mathrm{m} / \mathrm{z}$ values of molecular ion peaks in the

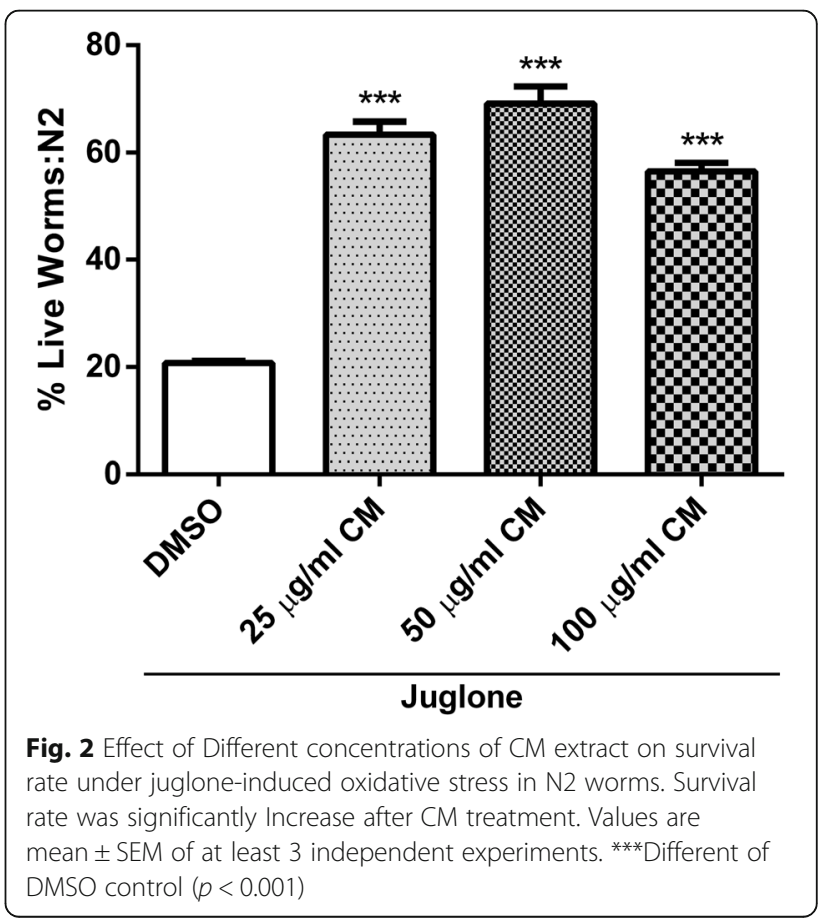



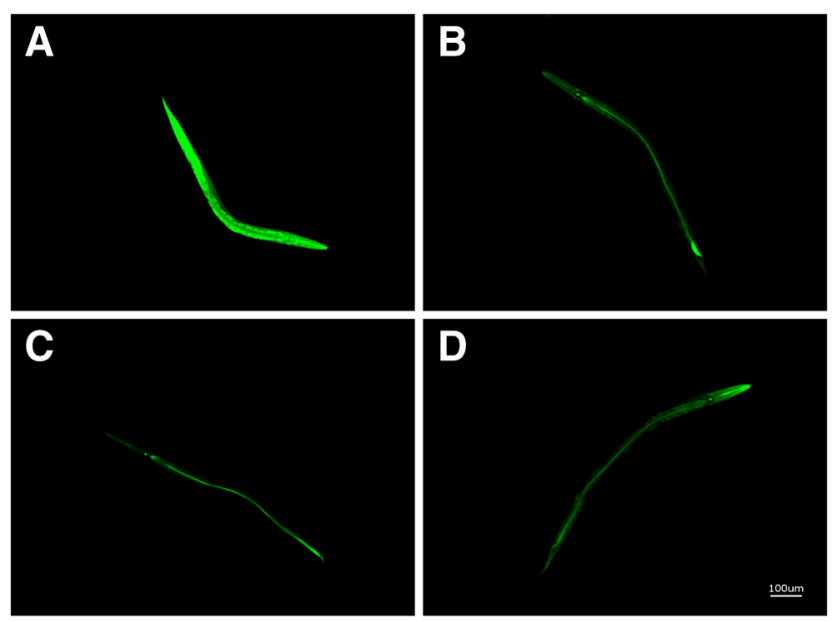

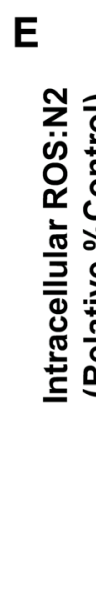

Fig. 3 Effect of CM extracts on intracellular ROS accumulation. N2 worms were treated with DMSO (a) and CM extracts 25,50 and $100 \mu \mathrm{g} / \mathrm{ml}$ (bd, respectively) incubated with H2DCF-DA for measurement of intracellular ROS accumulation. Intracellular ROS was significantly decrease after CM treatment (e). Values are mean \pm SEM of at least 3 independent experiments. ${ }^{* *}$ Different of DMSO control $(p<0.001)$

positive mode $[\mathrm{M}+\mathrm{H}]^{+}$. The five major phytochemical compounds were 3-O-methylgallate, 4-aminomethylindole, emmotin A, theogallin and gallic acid (Table 1).

\section{Total phenolic and flavonoid contents}

The total phenolic and flavonoid contents in extracts from young twig and leaves of C. mimosoides (CM) were determined. The highest total phenolic $(460.25 \pm 3.08 \mathrm{mg}$ GAE/g dry weight extract) and total flavonoid (12.55 \pm $0.43 \mathrm{mg} \mathrm{QE} / \mathrm{g}$ dry weight extract) contents were found in the methanol extract, followed by the ethyl acetate extract and then the hexane extract (Table 2). Due to these promising results, the $\mathrm{CM}$ methanol extract was therefore selected for subsequent experiments.
In vitro evaluation of antioxidant properties

DPPH and ABTS assays were used to investigate the free radical scavenging capacities of $\mathrm{CM}$ extracts in vitro. Methanol extracts possessed strong antioxidant activities because they exhibited high scavenging activities against DPPH and ABTS radicals. The effective concentration (EG50) was recorded (Table 3).

Effect of CM extract against juglone-induced oxidative stress in wild type worms

Juglone, a yellow pigmented pro-oxidant from Juglans regia, is commonly used to induce ROS-related mortality of C. elegans [25]. Only $20 \%$ of the worms survived after being treated with $80 \mu \mathrm{M}$ juglone. However, pretreatment
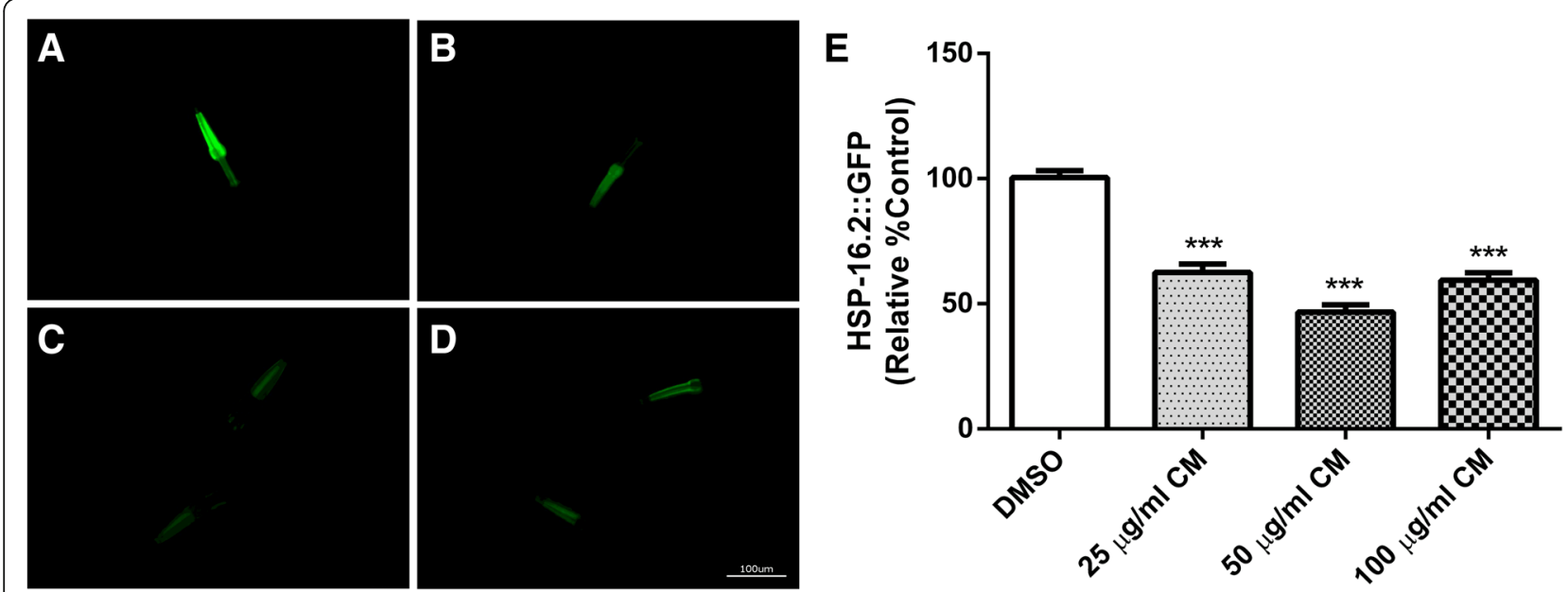

Fig. 4 To assessment of HSP-16::GFP expression after CM treatment. TJ375 worms were treated with DMSO (a) and CM extracts 25, 50 and $100 \mathrm{\mu g} / \mathrm{ml}$ (b-d, respectively). The expression of HSP::GFP significantly decrease after CM treatment (e). Values are mean \pm SEM of at least 3 independent experiments. ${ }^{* *}$ Different of DMSO control $(p<0.001)$ 


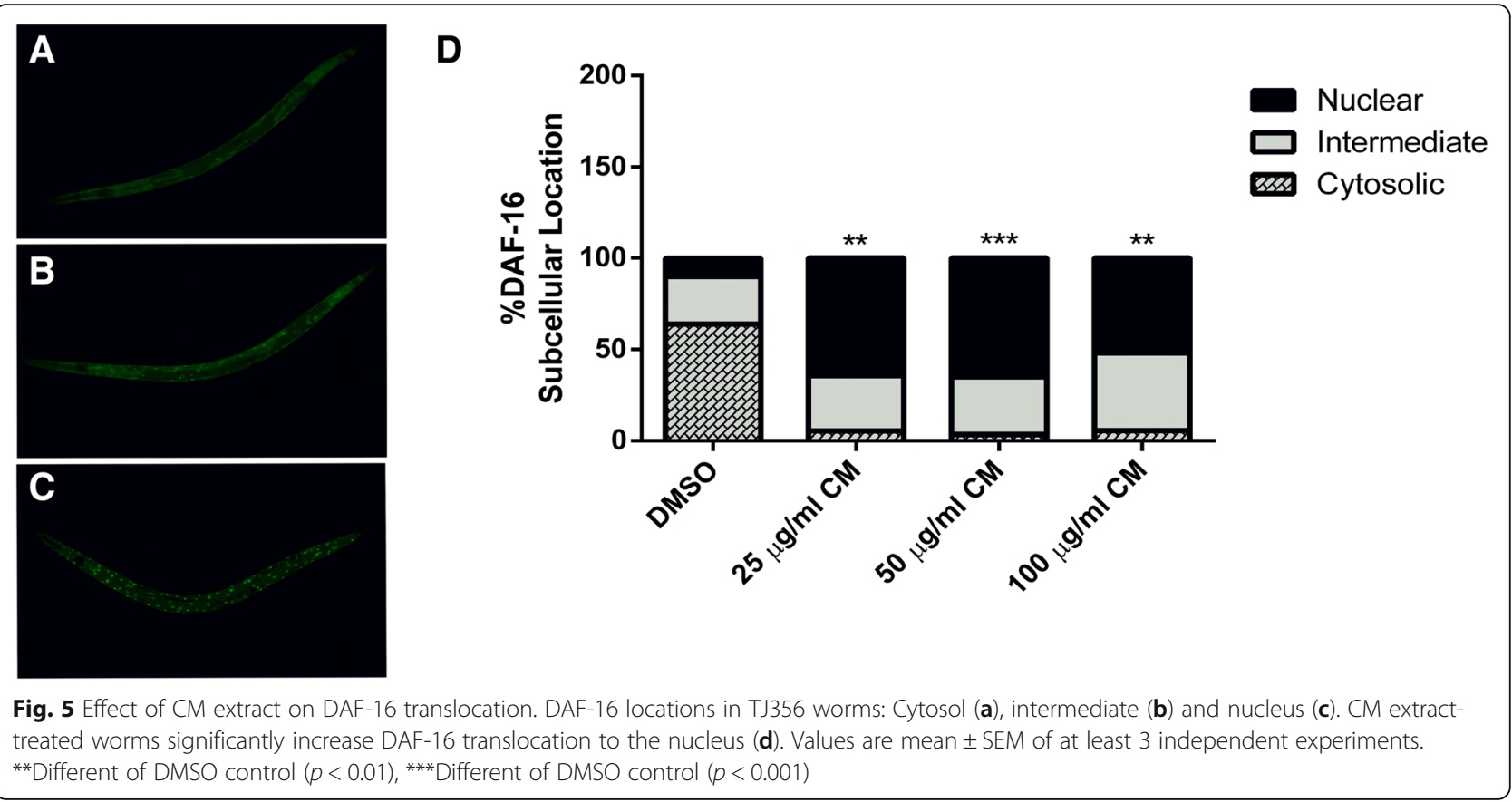

of the worms with methanol extracts $(25,50$ and $100 \mu \mathrm{g} /$ $\mathrm{ml}$ ) significantly enhanced the survival rates. Among the tested concentrations, $50 \mu \mathrm{g} / \mathrm{ml}$ extract showed the highest survival percentage $(69.11 \pm 2.36 \%)$ when compared to the DMSO solvent control $(20.79 \pm 0.38 \%) \quad(p<0.001)$ (Fig. 2).

Effect of CM extract on intracellular ROS accumulation in wild type worms

To further test the antioxidant effect of the methanol extract in vivo, intracellular ROS levels were evaluated in wild type $\mathrm{N} 2$ worms using $\mathrm{H}_{2}$ DCF-DA, a widely known fluorescence probe for detecting intracellular ROS production. The ROS level correlates with the fluorescence intensity resulting from oxidation by ROS, leading to the formation of the highly fluorescent $2^{\prime} 7$ '-dichlorofluorescin [26]. The results showed a significant decrease in the fluorescence intensity of the extract-treated groups $(25$, 50 and $100 \mu \mathrm{g} / \mathrm{ml}$ ), and the lowest levels were in the group treated with $50 \mu \mathrm{g} / \mathrm{ml}$ extract compared to the DMSO control group $(p<0.001)$ (Fig. 3). $100 \mu \mathrm{g} / \mathrm{ml}$ showed some pro-oxidant activity.
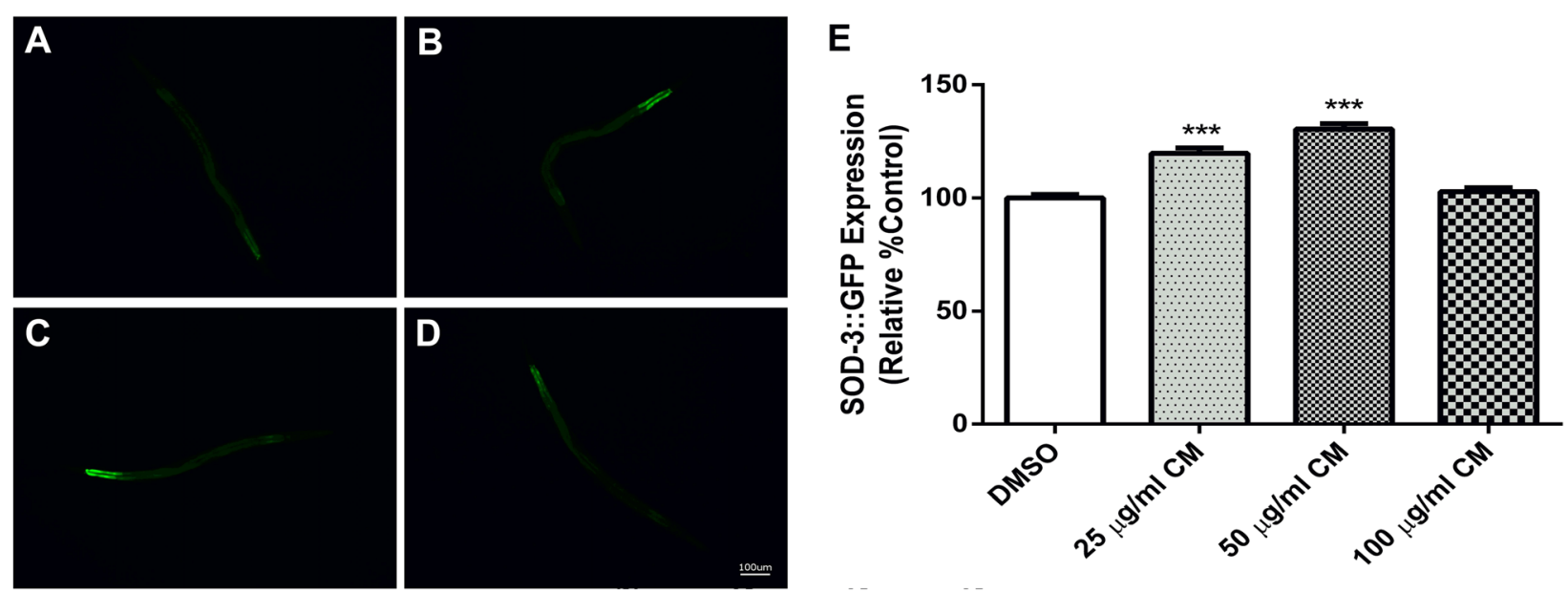

Fig. 6 Effect of CM extracts on SOD-3::GFP expression. CF1553 worms were treated with DMSO (a) and CM extracts 25, 50 and $100 \mu \mathrm{mg} / \mathrm{ml}$ (b-d, respectively). The expression of SOD-3:GFP significantly enhance after CM treatment (e). Values are mean \pm SEM of at least 3 independent experiments. ${ }^{* * *}$ Different of DMSO control $(p<0.001)$ 

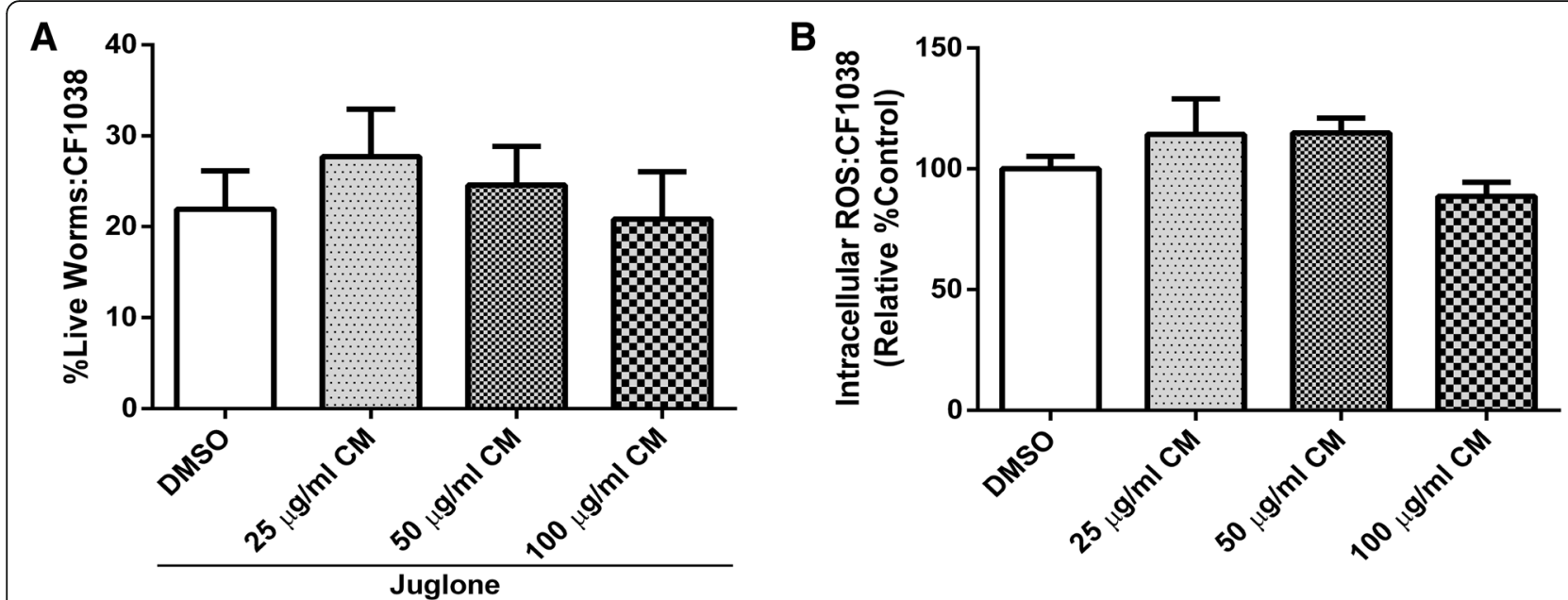

Fig. 7 CF1038 (DAF-16 loss of function) worms were used to confirm the effect of CM extract on DAF-16/FOXO pathway by survival assay under juglone-induced oxidative stress (a) and Intracellular ROS measurement (b) which the worms failed to counteract with stress and reduce intracellular ROS, respectively

\section{Effect of CM extract on HSP-16.2 expression}

TJ375 mutant worms express heat shock protein (HSP)16.2 , which is commonly used as a marker of oxidative stress in the nematode $[27,28]$. To further test the antioxidant effect of the methanol extract, HSP-16.2 levels were investigated in the worms treated with juglone and the extract. The expression of HSP-16.2 was significantly reduced in worms that were pretreated with the extract $(25,50$ and $100 \mu \mathrm{g} / \mathrm{ml})$ compared to the expression in worms treated with $20 \mu \mathrm{M}$ juglone alone. Among the concentrations tested, the $50 \mu \mathrm{g} / \mathrm{ml}$ extract treatment showed the lowest expression of HSP-16.2 (46.63 \pm $5.93 \%)$ when compared to the DMSO control group $(p<0.001)$ (Fig. 4).

\section{Effect of CM extract on DAF-16/FOXO pathway}

To investigate the mechanisms involved in the antioxidant effect of CM, the expression of transcription factor DAF-16, the C. elegans homologue to the fork head transcription factor (FOXO) in humans, was examined in TJ356 transgenic worms. Treatment with the extract (25, 50 and $100 \mu \mathrm{g} / \mathrm{ml}$ ) enhanced DAF-16 translocation from the cytoplasm to the nucleus. The $50 \mu \mathrm{g} / \mathrm{ml}$ extract resulted in a high percentage of nuclear subcellular localization of DAF-16::GFP (65.02 $\pm 4.55 \%)$ compared to the DMSO control group $(9.96 \pm 2.97 \%)(p<0.001)$. This result suggests that the antioxidant effect of the extract was mediated through the DAF-16/FOXO pathway (Fig. 5).

Normally, DAF-16 activation subsequently results in the activation of other stress response genes, such as SOD-3, which is a key enzyme that protects the worms against ROS [29]. We found that SOD-3:GFP showed higher expression levels in CF1553 transgenic worms treated with 25 and $50 \mu \mathrm{g} / \mathrm{ml}$ extract $(119.8 \pm 2.315$ and $130.5 \pm 2.392$, respectively) compared to the DMSO control group $(p<0.001)$. However, $100 \mu \mathrm{g} / \mathrm{ml}$ of the extract did not show any difference in fluorescence intensity compared to the control (Fig. 6).
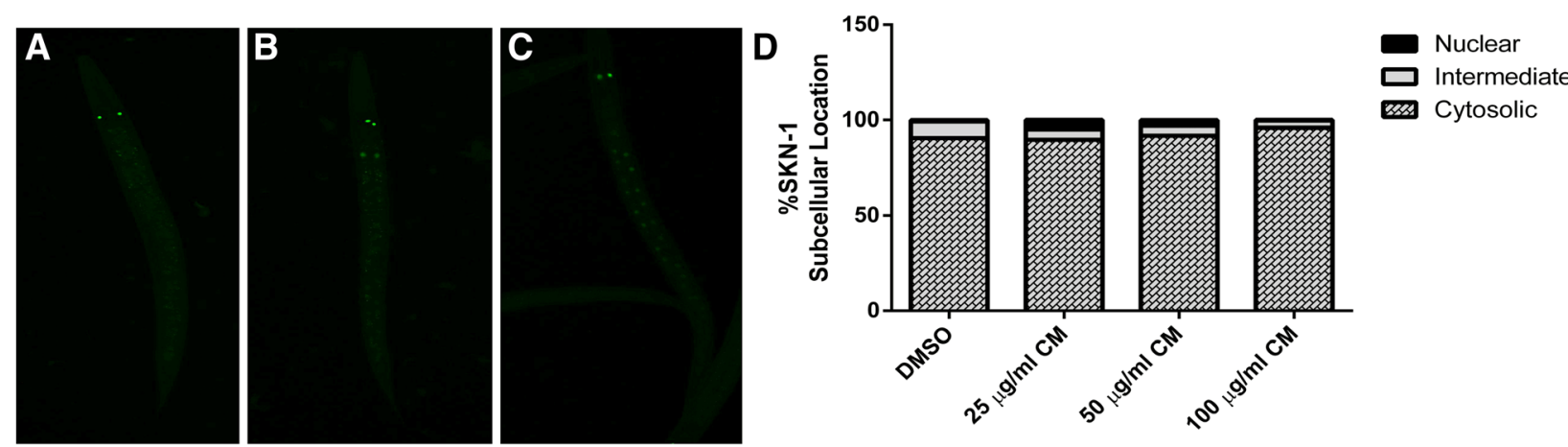

Fig. 8 Effect of SKN-1 translocation after treatment with CM extracts. SKN-1 locations in LD1 worms: Cytosol (a), intermediate (b) and nucleus (c). No alteration of SKN-1 subcellular localization was observed (d). Values are mean \pm SEM of at least 3 independent experiments 


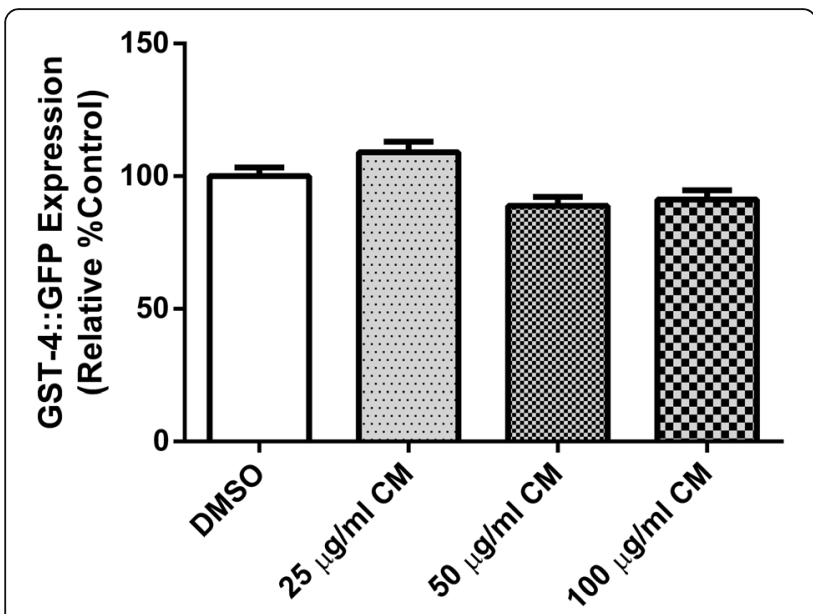

Fig. 9 Effect of CM extracts on GST-4::GFP expression. CL2166 worms were treated with DMSO and CM extracts 25, 50 and $100 \mu \mathrm{g} /$ $\mathrm{ml}$ for measurement of GST-4:.GFP expression (E). Values are mean \pm SEM of at least 3 independent experiments

To further confirm the antioxidant effect of $\mathrm{CM}$ via the DAF-16/FOXO pathway, DAF-16 loss-of-function transgenic worms CF1038 were employed to examine their survival and intracellular ROS levels under juglone-induced oxidative stress. Extract-treated DAF-16 mutant worms were unable to compensate for the mortality under juglone-induced oxidative stress. Likewise, no difference of intracellular ROS accumulation between CM extracts and DMSO control groups was detected (Fig. 7).

\section{Effect of CM extract on SKN-1/NRF-2 pathway}

$\mathrm{SKN}-1$, the $C$. elegans homologue to the mammalian NRF2 transcription factors, is known as a major regulator

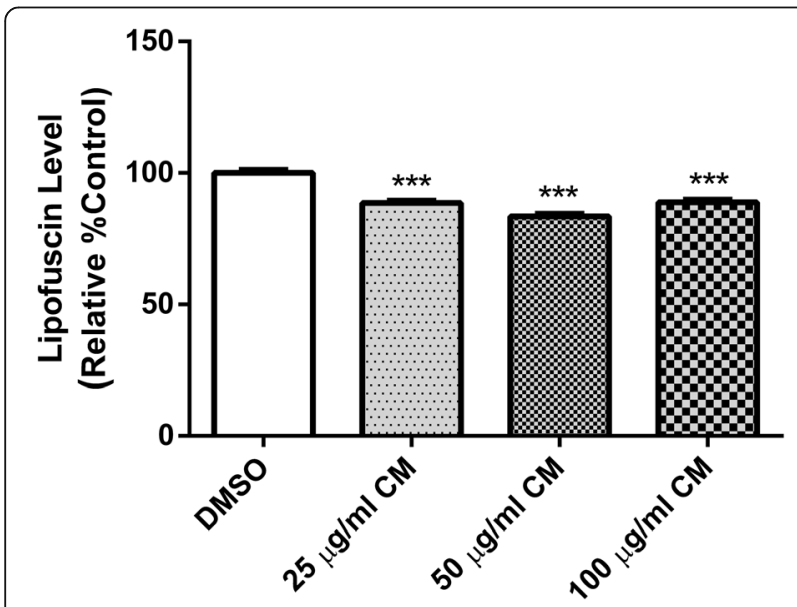

Fig. 10 Effect of CM extract on aging pigment lipofuscin level. BA17 worms were treated with DMSO and CM extracts 25,50 and $100 \mu \mathrm{g} /$ $\mathrm{ml}$. CM-treated worms significantly attenuate lipofuscin level. Values are mean \pm SEM of at least 3 independent experiments. ${ }^{* *}$ Different of DMSO control $(p<0.001)$ of antioxidant response in C. elegans. LD-1 transgenic worms, treated with 25,50 and $100 \mu \mathrm{g} / \mathrm{ml}$ extracts did not show any difference in SKN-1 nuclear translocation when compared to DMSO control group (Fig. 8).

GST-4 is an isoform of the glutathione S-transferases, which are involved in the worm's response to oxidative stress. This gene is regulated by the SKN-1 transcription factor. These results support the SKN-1 experiments (Fig. 8) in that extracts $(25,50$ and $100 \mu \mathrm{g} / \mathrm{ml})$ had no effect on the expression of GST-4 in CL2166 worms when compared to the DMSO control (Fig. 9).

\section{Effect of CM extract on lipofuscin level}

Intestinal cells of $C$. elegans contain lysosomes and gut granules called lipofuscin, which are autofluorescent. Lipofuscin accumulation increases during oxidative stress and aging. The expression of lipofuscin was reduced in the BA17 transgenic worms treated with methanol extract $(25,50$ and $100 \mu \mathrm{g} / \mathrm{ml})$. The extract treatment at a concentration of $50 \mu \mathrm{g} / \mathrm{ml}$ showed the highest reduction of autofluorescence $(16.60 \pm 1.10 \%)$ when compared to the DMSO control group $(p<0.001)$ (Fig. 10).

\section{Effect of CM extract on lifespan extension}

To evaluate anti-aging properties, we investigated the effect of CM extract on the lifespan of wildtype nematodes under normal conditions. The results showed that the extract at a concentration of $50 \mu \mathrm{g} / \mathrm{mL}$ was capable of enhancing the survival of wildtype N2 worms when compared to the DMSO control. The mean lifespan of the $50 \mu \mathrm{g} / \mathrm{ml}$ extract-treated L4-stage worms was $12.95 \mathrm{~d}$, which was slightly longer than that of the control group, with a significant difference at $p<0.001$ (Fig. 11 and Table 4).

\section{Effect of CM extract on body length and brood size}

To investigate the toxicity of the methanol extract, body length and brood size assays were performed to examine the development and fertility rate of the worms, respectively. These analyses could also be indicative of dietary restriction, which could also influence longevity. Analyses of body length revealed no difference in mean body length in worms treated with 25, 50 and $100 \mu \mathrm{g} / \mathrm{ml}$ extract when compared with the DMSO control (Fig. 12a). Additionally, the brood size assay did not show alterations in the number of eggs laid after extract treatment (Fig. 12b). Thus, dietary restriction can be ruled out.

\section{Discussion}

Our study confirms previous reports that the methanol extract of $C$. mimosoides (CM) is rich in phenolics and flavonoids. The methanol extract exhibited the highest free radical scavenging capacity in vitro via DPPH and 


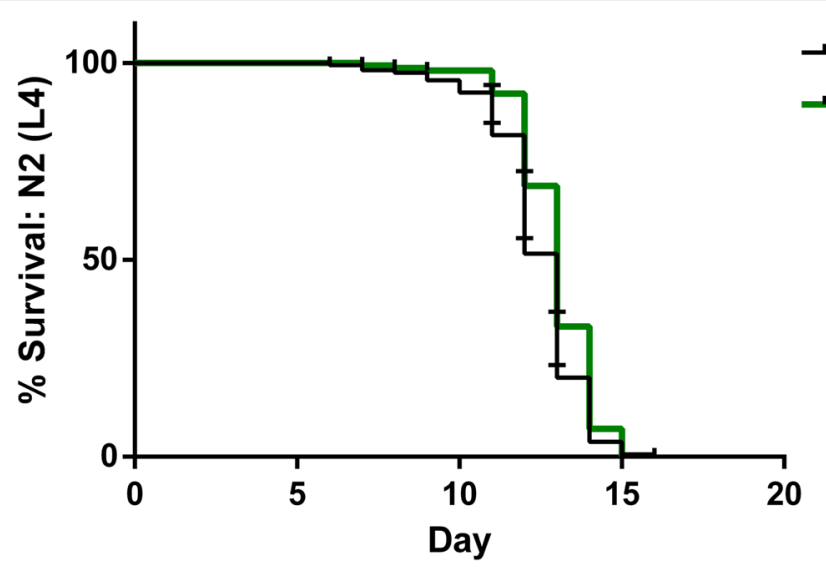

Fig. 11 Longevity of N2 worm-treated with CM extracts. Lifespan extension after $50 \mu \mathrm{g} / \mathrm{ml}$ of CM extract treatment showed in cumulative survival plots

ABTS assays compared with hexane and ethyl acetate extracts. Similarly, previous studies of CM have obtained bioactive compounds from the polar substances rather than the nonpolar substances [8-11,30,31]. The major phenolic compounds of the extract identified by LC-MS in our study include gallic acid, theogallin, bergenin, 3-O-methylgallate, quercetin, clausarinol and emmotin A. This finding is similar to previous reports that gallic acid is a major constituent of the compounds in CM extract [10,11,31].

To further investigate a potential antioxidant activity of the methanol extract in vivo, C. elegans was employed as a model organism. The extract increased survival rates in $C$. elegans under juglone-induced oxidative stress and lowered intracellular ROS levels. In addition, HSP-16.2 was also significantly lower in CM-treated worms than in the control. Taken together, these results supported that the phenolics of $\mathrm{CM}$ are effective antioxidants in vivo and that they are bioavailable. These findings agree with several other studies that clearly showed an antioxidant effect of polyphenols [17-19, 32-34].

To investigate the mechanism underlying the antioxidant effect of CM extract, DAF-16/FOXO and SKN-1/ NRF-2 pathways were monitored in this study. Normally, the transcription factor DAF-16 is localized in the cytosol in its inactive phosphorylated form. Oxidative damage can induce its activation by dephosphorylation and subsequent translocation into the nucleus. DAF-16 activation is responsible for stress response and lifespan extension [35]. Upon nuclear localization, DAF-16 induces the transcription of several genes involving antioxidant systems, such as SOD-3, which encodes mitochondrial superoxide dismutase (Mn-SOD). Previous studies suggested that this enzyme could protect the worms against ROS via elimination of free radicals [29]. The transcription factor SKN-1 regulates the expression of downstream genes of phase II detoxification enzymes. [36] GST-4 is an isoform of glutathione S-transferases that plays a major role in phase II detoxification process in C. elegans and can be activated by SKN-1. [37]

In the present study, we observed enhanced translocation of DAF-16 after treatment, whereas the localization of SKN-1 was not affected. The expression of SOD-3 and GST-4 genes were altered accordingly. Therefore, these results suggest that the extract exerts its antioxidant effect through the activation of DAF-16/FOXO pathway. Moreover, our findings are consistent with previous observations with polyphenol-rich plant extracts and isolated compounds that protect $C$. elegans against oxidative stress via the DAF-16/FOXO pathway [2, 19, 38-42].

Elevated ROS production apparently is a major contributing factor in the aging process [43]. Lipofuscin, an indicator of both oxidative stress and aging in C. elegans [44], is an autofluorescent pigment that accumulates progressively over time, particularly in lysosomes and gut granules of intestinal [45]. We found a lower accumulation of lipofuscin after CM treatment, which is in agreement with the observed antioxidant and antiaging capacities of the extract. [18, 33, 34, 39, 46] Lifespan was slightly enhanced by the extract; this effect was not due to dietary restriction because neither the

Table 4 Results and statistical analyses of C. elegans lifespan assay

\begin{tabular}{|c|c|c|c|c|c|}
\hline Treatment: N2 (L4) & Mean lifespan (day) \pm SEM & \% increased lifespan (vs. control) & $p$-value (vs. control) & $p$-value summary & Number of worms \\
\hline Control & $12.41 \pm 0.12$ & & & & $N=159$ \\
\hline $50 \mu \mathrm{g} / \mathrm{ml} \mathrm{CM}$ extract & $12.95 \pm 0.10$ & 4.35 & 0.0003 & $* * *$ & $N=154$ \\
\hline
\end{tabular}

The life span assay was carried out with wild type (N2) worms at $25^{\circ} \mathrm{C}$. Mean lifespan in days is the average number of days the worms survived in each group. The treatment group was compared to the control by log-rank (Mantel - Cox) tests followed by the Gehan - Breslow - Wilcoxon test 

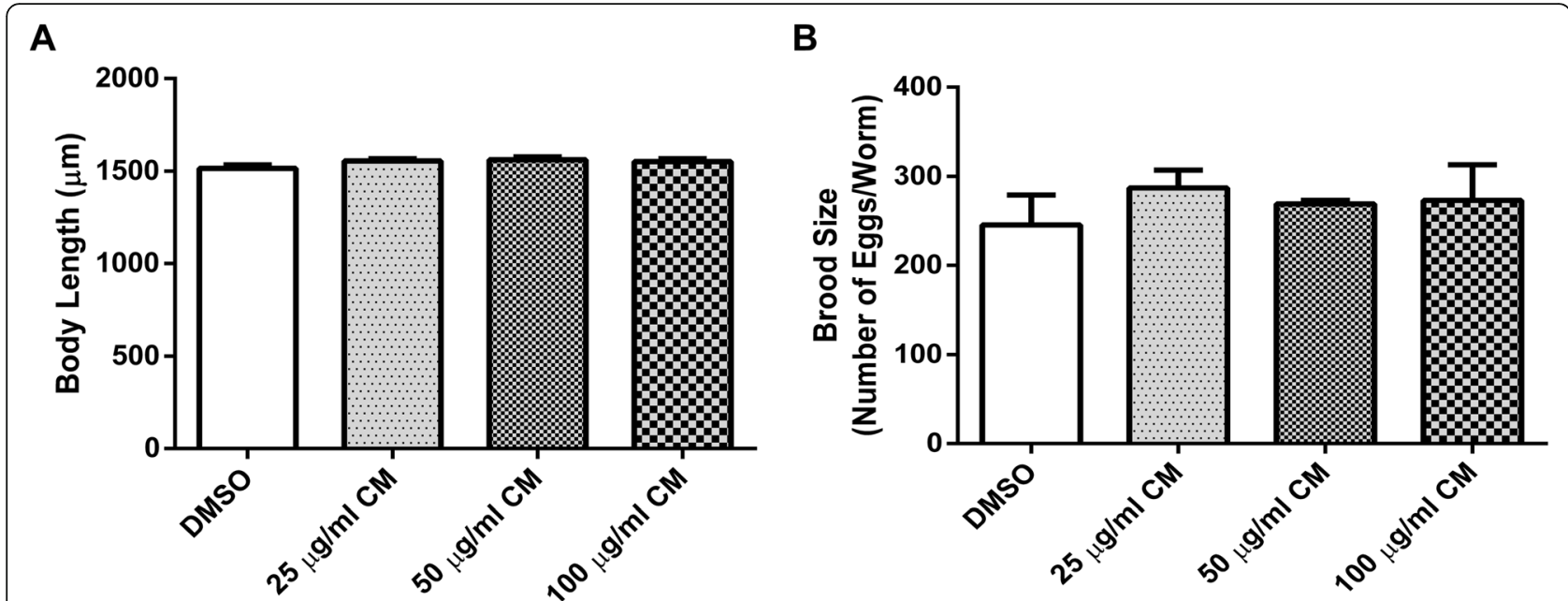

Fig. 12 Effect of CM extracts on body length (a) and brood size (b) in N2 worms. Values are mean \pm SEM of at least 3 independent experiments

development nor the fertility of the worms were impaired.

\section{Conclusions}

In conclusion, the current study demonstrates the relevant antioxidant and anti-aging activities of the CM extract in $C$. elegans. The extract was able to increase stress resistance and to reduce intracellular ROS levels as well as the expression of the HSP stress gene after exposure to oxidative stress. The extract was also able to enhance the nuclear localization of the DAF-16 transcription factor and the expression of the SOD-3 gene, demonstrating that its antioxidant activity is probably mediated through the DAF16/ FOXO pathway. Overall, these results suggest that $C$. mimosoides could be a potential dietary supplement and alternative medicine with antioxidant and anti-aging properties. However, intervention studies with other organisms are required to corroborate our findings in C. elegans.

\section{Additional file}

Additional file 1: Antimicrobial activity test using agar diffusion method. CM extract at the concentration $200 \mu \mathrm{g} / \mathrm{ml}$ showed no effect on E. coli OP50 (PDF $68 \mathrm{~kb}$ )

\section{Abbreviations}

ABTS: 2,2-Azino-bis (3-ethylbenzothiazoline-6-sulfonic acid); CM: Caesalpinia mimosoides L; DAF-16/FOXO: Forkhead box protein O; DMSO: Dimethyl sulfoxide; DPPH: Diammonium salt, 2,2-Diphenyl-1-picrylhydrazyl; EGCG: Epigallocatechin gallate; GAE: Gallic acid equivalents; GFP: Green fluorescence protein; GST-4: Glutathione S-transferase 4; H2DCF-DA: 2,7dichlorofluorescein diacetate; HSP-16.2: Heat shock protein-16.2; Juglone: (5Hydroxy-1,4-naphthoquinone); LC-MS: Liquid chromatography-mass spectrometry; $\mathrm{NaOCl}$ : Sodium hypochlorite; $\mathrm{NaOH}$ : Sodium hydroxide; QE: Quercetin equivalents; ROS: Reactive oxygen species; SKN-1/Nrf2: Nuclear factor erythroid 2-related factor 2; SOD-3: Superoxide dismutase-3

\section{Acknowledgements}

The C. elegans experiments were carried out at Heidelberg University (Germany). Special thanks to Mariana Roxo and Pille Link (IPMB, Heidelberg
University, Germany) for instructions on C. elegans experiments, and Prof. Charles Henry (Colorado State University, CO, USA) for providing language help and proof reading the article.

\section{Authors' contributions}

PR performed the C. elegans experiments, analyzed data, and was a major contributor in writing the manuscript. AP performed plant extraction, in vitro study and preliminary study in C. elegans. CD took photos of C. elegans. PR, $C D$ and $X G$ designed the study and prepared media and reagents. MW and KM provided materials for the study in C. elegans. MW and TT conceived and supervised research. MW, TT, AP, XG and KM corrected the manuscript. All authors have read and approved the final version of the manuscript.

\section{Funding}

Panthakarn Rangsinth was financially supported by a scholarship from "The $100^{\text {th }}$ Anniversary Chulalongkorn University Fund for Doctoral Scholarship" and "The $90^{\text {th }}$ Anniversary Chulalongkorn University Fund

(Ratchadaphiseksomphot Endowment Fund)" funding code

GCUGR1125612058D No. Forty-nine for research expense and also "The Overseas Research Experience Scholarship for Graduate Students" from Graduate School, Chulalongkorn University for the research visit in Germany.

Availability of data and materials

All data generated or analysed during this study are included in this published article. The datasets used and/or analysed during the current study available from the corresponding author on reasonable request.

Ethics approval and consent to participate

Not applicable.

\section{Consent for publication}

Not applicable.

\section{Competing interests}

The authors declared that they have no competing interests.

\section{Author details}

${ }^{1}$ Graduate Program in Clinical Biochemistry and Molecular Medicine, Department of Clinical Chemistry, Faculty of Allied Health Sciences, Chulalongkorn University, Bangkok 10330, Thailand. ${ }^{2}$ College of Public Health Sciences, Chulalongkorn University, Bangkok 10330, Thailand. ${ }^{3}$ Department of Biotechnology, School of Environmental and Chemical Engineering, Dalian Jiaotong University, Dalian 116028, China. ${ }^{4}$ Department of Anatomy, Faculty of Science, Mahidol University, Bangkok 10400, Thailand. ${ }^{5}$ Institute of Pharmacy and Molecular Biotechnology, Heidelberg University, Im Neuenheimer Feld 364, 69120 Heidelberg, Germany. ${ }^{6}$ Age-Related Inflammation and Degeneration Research Unit, Department of Clinical 
Chemistry, Faculty of Allied Health Sciences, Chulalongkorn University, Bangkok 10330, Thailand.

Received: 15 April 2019 Accepted: 26 June 2019

Published online: 08 July 2019

\section{References}

1. Fang $Y Z$, Yang $S$, Wu G. Free radicals, antioxidants, and nutrition. Nutrition (Burbank, Los Angeles County, Calif). 2002;18(10):872-9.

2. Tambara AL, De Los Santos Moraes L, Dal Forno AH, Boldori JR, Goncalves Soares AT, De Freitas Rodrigues C, et al. Purple Pitanga fruit (Eugenia uniflora L.) protects against oxidative stress and increase the lifespan in Caenorhabditis elegans via the DAF-16/FOXO pathway. Food and chemical toxicology : an international journal published for the British Industrial Biological Research Association. 2018:120:639-50.

3. Liu Z, Ren Z, Zhang J, Chuang CC, Kandaswamy E, Zhou T, et al. Role of ROS and Nutritional antioxidants in human diseases. Front Physiol. 2018:9:477.

4. Finkel T, Holbrook NJ. Oxidants, oxidative stress and the biology of ageing. Nature. 2000;408(6809):239-47.

5. Yu BP. Cellular defenses against damage from reactive oxygen species Physiol Rev. 1994;74(1):139-62.

6. Fridovich I. Fundamental aspects of reactive oxygen species, or what's the matter with oxygen? Ann N Y Acad Sci. 1999;893:13-8.

7. Liguori I, Russo G, Curcio F, Bulli G, Aran L, Della-Morte D, et al. Oxidative stress, aging, and diseases. Clin Interv Aging. 2018;13:757-72.

8. Chanwitheesuk A, Teerawutgulrag A, Rakariyatham N. Screening of antioxidant activity and antioxidant compounds of some edible plants of Thailand. Food Chem. 2005:92(3):491-7.

9. Yodsaoue O, Karalai C, Ponglimanont C, Tewtrakul S, Chantrapromma S. Potential anti-inflammatory diterpenoids from the roots of Caesalpinia mimosoides Lamk. Phytochemistry. 2010;71(14-15):1756-64.

10. Rattanata N, Klaynongsruang S, Daduang S, Tavichakorntrakool R, Limpaiboon T, Lekphrom R, et al. Inhibitory effects of Gallic acid isolated from Caesalpinia mimosoides Lamk on cholangiocarcinoma cell lines and foodborne pathogenic Bacteria. Asian Pacific journal of cancer prevention APJCP. 2016;17(3):1341-5.

11. Chanwitheesuk A, Teerawutgulrag A, Kilburn JD, Rakariyatham N. Antimicrobial gallic acid from Caesalpinia mimosoides Lamk. Food Chem. 2007;100(3):1044-8.

12. Shankar S, Tanomrod N, Rawdkuen S, Rhim JW. Preparation of pectin/silver nanoparticles composite films with UV-light barrier and properties. Int J Biol Macromol. 2016;92:842-9.

13. Partridge L, Gems D. Beyond the evolutionary theory of ageing, from functional genomics to evo-Gero. Trends Ecol Evol. 2006;21(6):334-40.

14. Genome sequence of the nematode. C. elegans: a platform for investigating biology. Science (New York, NY). 1998;282(5396):2012-2018

15. Hillier LW, Coulson A, Murray Jl, Bao Z, Sulston JE, Waterston RH. Genomics in C. elegans: so many genes, such a little worm. Genome Res. 2005;15(12):1651-60.

16. Harris TW, Chen N, Cunningham F, Tello-Ruiz M, Antoshechkin I, Bastiani C, et al. WormBase: a multi-species resource for nematode biology and genomics. Nucleic Acids Res. 2004;32(Database issue):D411-7.

17. Kampkotter A, Timpel C, Zurawski RF, Ruhl S, Chovolou Y, Proksch P, et al. Increase of stress resistance and lifespan of Caenorhabditis elegans by quercetin. Comparative biochemistry and physiology Part B, Biochemistry \& molecular biology. 2008;149(2):314-23.

18. Abbas S, Wink M. Epigallocatechin gallate from green tea (Camellia sinensis) increases lifespan and stress resistance in Caenorhabditis elegans. Planta Med. 2009;75(3):216-21.

19. Peixoto H, Roxo M, Krstin S, Rohrig T, Richling E, Wink M. An anthocyaninrich extract of acai (Euterpe precatoria Mart.) increases stress resistance and retards aging-related markers in Caenorhabditis elegans. J Agric Food Chem. 2016;64(6):1283-90.

20. Chen J, Zhang J, Xiang Y, Xiang L, Liu Y, He X, et al. Extracts of Tsai tai (Brassica chinensis): enhanced antioxidant activity and anti-aging effects both in vitro and in Caenorhabditis elegans. Food Funct. 2016;7(2):943-52.

21. Prasansuklab A, Meemon K, Sobhon P, Tencomnao T. Ethanolic extract of Streblus asper leaves protects against glutamate-induced toxicity in HT22 hippocampal neuronal cells and extends lifespan of Caenorhabditis elegans. BMC Complement Altern Med. 2017;17(1):551.

22. Prasansuklab A, Theerasri A, Payne M, Ung AT, Tencomnao T. Acid-base fractions separated from Streblus asper leaf ethanolic extract exhibited antibacterial, antioxidant, anti-acetylcholinesterase, and neuroprotective activities. BMC Complement Altern Med. 2018;18(1):223.

23. Prasansuklab A, Tencomnao T. Acanthus ebracteatus leaf extract provides neuronal cell protection against oxidative stress injury induced by glutamate. BMC Complement Altern Med. 2018;18(1):278.

24. Thabit S, Handoussa H, Roxo M, El Sayed NS, Cestari de Azevedo B, Wink M. Evaluation of antioxidant and neuroprotective activities of Cassia fistula (L.) using the Caenorhabditis elegans model. PeerJ. 2018;6:e5159.

25. Inbaraj JJ, Chignell CF. Cytotoxic action of juglone and plumbagin: a mechanistic study using HaCaT keratinocytes. Chem Res Toxicol. 2004;17(1):55-62.

26. Eruslanov E, Kusmartsev S. Identification of ROS using oxidized DCFDA and flow-cytometry. Methods in molecular biology (Clifton, NJ). 2010;594:57-72.

27. Strayer $A, W u Z$, Christen Y, Link CD, Luo Y. Expression of the small heatshock protein Hsp16-2 in Caenorhabditis elegans is suppressed by Ginkgo biloba extract EGb 761. FASEB journal : official publication of the Federation of American Societies for Experimental Biology. 2003;17(15):2305-7.

28. Swindell WR. Heat shock proteins in long-lived worms and mice with insulin/insulin-like signaling mutations. Aging. 2009;1(6):573-7.

29. McElwee J, Bubb K, Thomas JH. Transcriptional outputs of the Caenorhabditis elegans forkhead protein DAF-16. Aging Cell. 2003;2(2):111-21.

30. Kim YJ. Antimelanogenic and antioxidant properties of gallic acid. Biol Pharm Bull. 2007:30(6):1052-5.

31. Palasap A, Limpaiboon T, Boonsiri P, Thapphasaraphong S, Daduang S, Suwannalert P, et al. Cytotoxic effects of Phytophenolics from Caesalpinia mimosoides Lamk on cervical carcinoma cell lines through an apoptotic pathway. Asian Pacific journal of cancer prevention : APJCP. 2014;15(1):449-54.

32. Abbas S, Wink M. Green tea extract induces the resistance of Caenorhabditis elegans against oxidative stress. Antioxidants (Basel, Switzerland). 2014;3(1):129-43.

33. Peixoto H, Roxo M, Koolen H. Calycophyllum spruceanum (Benth.), the Amazonian "Tree of Youth" Prolongs Longevity and Enhances Stress Resistance in Caenorhabditis elegans. 2018;23(3).

34. Peixoto $H$, Roxo M, Rohrig T, Richling E, Wang X. Anti-Aging and Antioxidant Potential of Paullinia cupana var. sorbilis: Findings in Caenorhabditis elegans Indicate a New Utilization for Roasted Seeds of Guarana. 2017:4(3).

35. Mukhopadhyay A, Oh SW, Tissenbaum HA. Worming pathways to and from DAF-16/FOXO. Exp Gerontol. 2006;41(10):928-34.

36. Kahn NW, Rea SL, Moyle S, Kell A, Johnson TE. Proteasomal dysfunction activates the transcription factor SKN-1 and produces a selective oxidative-stress response in Caenorhabditis elegans. The Biochemical journal. 2008:409(1):205-13.

37. Choe KP, Przybysz AJ, Strange K. The WD40 repeat protein WDR-23 functions with the CUL4/DDB1 ubiquitin ligase to regulate nuclear abundance and activity of SKN-1 in Caenorhabditis elegans. Mol Cell Biol. 2009;29(10):2704-15.

38. Rezaizadehnajafi L, Wink M. EPs7630((R)) from Pelargonium sidoides increases stress resistance in Caenorhabditis elegans probably via the DAF16/FOXO pathway. Phytomedicine : international journal of phytotherapy and phytopharmacology. 2014;21(4):547-50.

39. Chen W, Muller D, Richling E, Wink M. Anthocyanin-rich purple wheat prolongs the life span of Caenorhabditis elegans probably by activating the DAF-16/FOXO transcription factor. J Agric Food Chem. 2013;61(12):3047-53.

40. Wang E, Wink M. Chlorophyll enhances oxidative stress tolerance in Caenorhabditis elegans and extends its lifespan. PeerJ. 2016;4:e1879.

41. Zhu B, Yang P, Mammat N, Ding H, He J, Qian Y, et al. Aiweixin, a traditional Uyghur medicinal formula, protects against chromium toxicity in Caenorhabditis elegans. BMC Complement Altern Med. 2015;15:285.

42. Duangjan C, Rangsinth P, Gu X, Wink M, Tencomnao T. Lifespan extending and oxidative stress resistance properties of a leaf extracts from Anacardium occidentale L. in Caenorhabditis elegans. Oxidative Med Cell Longev. 2019;2019.

43. Kong Y, Trabucco SE, Zhang H. Oxidative stress, mitochondrial dysfunction and the mitochondria theory of aging. Interdiscip Top Gerontol. 2014;39:86-107.

44. Sohal RS, Brunk UT. Lipofuscin as an indicator of oxidative stress and aging. Adv Exp Med Biol. 1989;266:17-26; discussion 7-9.

45. Clokey GV, Jacobson LA. The autofluorescent "lipofuscin granules" in the intestinal cells of Caenorhabditis elegans are secondary lysosomes. Mech Ageing Dev. 1986;35(1):79-94.

46. Chen W, Rezaizadehnajafi L, Wink M. Influence of resveratrol on oxidative stress resistance and life span in Caenorhabditis elegans. J Pharm Pharmacol. 2013;65(5):682-8.

\section{Publisher's Note}

Springer Nature remains neutral with regard to jurisdictional claims in published maps and institutional affiliations. 